\title{
Exercício Físico como Tratamento na Osteoartrite de Quadril: uma Revisão de Ensaios Clínicos Aleatórios Controlados
}

\section{Exercise Therapy as a Treatment in Osteoarthritis of the Hip: a Review of Randomized Clinical Trials}

\author{
Natália Aquaroni Ricci(1), Ibsen Bellini Coimbra ${ }^{(2)}$
}

\section{RESUMO}

As funções do quadril são cruciais para a independência funcional de um indivíduo, motivo da grande repercussão da osteoartrite (OA) sobre as alterações nessa articulação. A OA de quadril é uma doença crônico-degenerativa e o tratamento atual detém-se em combater sua sintomatologia. Dentre os possíveis tratamentos conservadores, os exercícios têm sido indicados para a redução da dor e melhora funcional. O objetivo desta revisão sistemática é elucidar a função e efetividade dos exercícios físicos no tratamento conservador da OA de quadril. Para a realização do levantamento bibliográfico foram consultadas as bases de dados PUBMED, LILACS e MEDLINE referentes às publicações de artigos de ensaio clínico aleatório controlado dos últimos dez anos (julho/2005 - julho/1995), utilizando-se a relação entre as palavras-chave osteoarthritis, hip, exercise. Resultaram da busca, 18 artigos. Desses, dez continham os critérios para revisão na íntegra. Após análise do conteúdo dos dez estudos, foram identificadas três categorias: a) exercícios específicos para OA de quadril $(\mathrm{n}=3)$; b) exercícios para OA de quadril e joelho $(\mathrm{n}=4)$ e c) exercícios para pré e póscirurgia de artroplastia decorrente de $\mathrm{OA}(\mathrm{n}=3)$. Dos dez estudos analisados, três revelaram limitações dos benefícios dos exercícios nas amostras. Embora, a maioria dos estudos tenha demonstrado bons resultados com a prática de exercícios no tratamento da $\mathrm{OA}$ de quadril, ainda não é possível determinar o alcance deste tipo de terapêutica no quadro geral da doença e para sua progressão, tendo em vista a escassez de estudos e falta de especificidade das intervenções propostas.

Palavras-chave: osteoartrite, quadril, exercício, ensaio clínico aleatório controlado.

\begin{abstract}
Functions of the hip are crucial for the functional independence of an individual, being the reason of great repercussion of Osteoarthritis $(O A)$ on the alterations in this joint. Hip $O A$ is a chronic degenerative disease and the available treatment aims to alleviate its symptoms. Exercises, one of the conservative treatment possibilities, have been indicated for the reduction of pain and function improvement. The purpose of this systematic review is to clarify the function and effectiveness of physical exercises in the conservative treatment of hip $O A$. The review of the literature was based on PUBMED, LILACS and MEDLINE database consultation, searching for article publications of randomized clinical trials for the last ten years (July/2005 - July/1995), using the combination of the following keywords: osteoarthritis, hip, exercise. The search resulted in eighteen articles; ten of each followed given criteria and underwent complete revision. After analysis of the ten selected studies, three categories were identified: a) specific exercises for $O A$ of hip $(n=3)$; b) exercises for $O A$ of hip and knee $(n=4)$ and c) exercises for pre and post-surgery of total hip (n=3). Three studies have encountered limitations with the practice of exercises. Although, the majority of the articles have demonstrated good results of exercises in the hip OA treatment, it is still not possible to determine their actual extension in the clinical picture of the illness and on its progression, mainly due to the scarcity of researches and to the lack of specificity of the proposed interventions.
\end{abstract}

Keywords: osteoarthritis, hip, exercise therapy, randomized clinical trials.

\section{INTRODUÇÃO}

A osteoartrite (OA) é uma enfermidade crônico-degenerativa que promove alterações na cartilagem articular, sendo a doença musculoesquelética mais comum em todo

o mundo ${ }^{(1,2,3)}$. A Organização Mundial da Saúde estima que $25 \%$ dos indivíduos acima de 65 anos sofrem de dor e incapacidade associadas a esta doença ${ }^{(4)}$.

\footnotetext{
Trabalho realizado na Disciplina de Biologia do Envelhecimento do Programa de Pós-Graduação em Gerontologia da Universidade Estadual de Campinas (UNICAMP). Recebido em 24/03/06. Aprovado, após revisão, em 09/07/06.

1. Fisioterapeuta Mestranda pelo Programa de Pós-Graduação em Gerontologia da UNICAMP.

2. Professor Doutor, Coordenador da Reumatologia da Faculdade de Ciências Médicas da UNICAMP.

Endereço para correspondência: Natália Aquaroni Ricci, Rua Pacaembu, 257/602, CEP 18040-710, Sorocaba, SP, Brasil, telefone: (15) 3232-3632,

e-mail: natalia_ricci@hotmail.com
} 
Dentre as formas clínicas de OA, destacam-se a OA de quadril e joelho por serem particularmente mais incapacitantes, já que essas são as articulações que recebem todo o peso corporal ${ }^{(4,5)}$. A OA de quadril atinge $20 \%$ das pessoas com idade acima de 55 anos $^{(6)}$ e, apesar de ser menos comum do que a OA de joelho, sua sintomatologia é freqüentemente mais grave ${ }^{(7)}$.

As funções do quadril são cruciais para a independência funcional de um indivíduo ${ }^{(8)}$, motivo da grande repercussão da OA sobre as alterações nessa articulação. A gravidade da OA de quadril leva a conseqüências que excedem a degeneração da cartilagem, estando diretamente relacionada com dor, rigidez articular e disfunção muscular ${ }^{(6)}$.

Por seu caráter crônico, o tratamento atual da $\mathrm{OA}$ de quadril detém-se em combater sua sintomatologia ${ }^{(9)}$. Assim, a prevenção e controle da progressão dos sintomas podem ser feitos com analgésicos, fisioterapia (analgesia e exercícios) e cirurgia de reposição articular (artroplastia de quadril - ATQ $)^{(10)}$. No entanto, nem todos os pacientes terão que passar pelo tratamento cirúrgico, além deste representar um alto custo pessoal e social ${ }^{(6)}$. Assim a alternativa para a maioria dos pacientes com OA é o tratamento conservador, que ajuda a reduzir e aliviar os sintomas, melhora a função e retarda a progressão, além de ser economicamente mais vantajoso ${ }^{(11)}$.

Entre os possíveis tratamentos incruentos da OA, admite-se os exercícios como uma possibilidade terapêutica apesar de não haver suporte técnico-científico de seus benefícios. No entanto, é possível visualizar que para as conseqüências da OA nas articulações dos membros inferiores, em especial na articulação coxofemoral, os exercícios e a atividade física têm um efeito positivo, enquanto a inatividade ou imobilidade contribuem para a progressão da doença ${ }^{(12)}$.

São poucos os estudos na literatura sobre a eficácia dos exercícios na OA de quadril, diferentemente da OA de joelho, em que já há demonstração de bons resultados deste tipo de intervenção ${ }^{(10)}$. Nas mais recentes revisões sistemáticas sobre o assunto ${ }^{(13,14)}$, foram analisadas juntamente a OA de quadril e joelho. No entanto, nenhuma conclusão pôde ser feita sobre os benefícios na OA de quadril, pois foram poucos os estudos randomizados que abordavam o acometimento nesta articulação. Além disso, o número de participantes com OA de quadril foi reduzido para a avaliação da dimensão dos efeitos dos exercícios na doença. Na busca à literatura, Roddy et a ${ }^{(15)}$ encontraram 159 artigos relacionados com terapia por exercício e OA de quadril ou joelho, sendo que, destes, apenas nove foram de OA de quadril, contra 57 de joelho. Enquanto Fransen et a ${ }^{(13)}$ encontraram quatro ensaios de
OA de quadril e 15 de joelho.

Os consensos sobre o manejo da OA, em geral, recomendam a terapia por exercício para a redução da dor e melhora funcional ${ }^{(16,17,18)}$. Assim, apesar de poucos critérios de evidência em pesquisa, este é um método que parece trazer bons resultados na prática clínica.

Embora reconhecida a função dos exercícios na redução da dor, aumento da força muscular, produção de estabilidade articular, aumento da flexibilidade e melhora da capacidade aeróbica ${ }^{(10)}$, ainda é necessário saber o alcance dessas mudanças no paciente com $\mathrm{OA}$ de quadril, quais e como os exercícios devem ser prescritos, quais exercícios devem ser evitados, resposta a curto e longo prazo, e a adesão dos pacientes a programas individuais e em grupo. Somente quando as respostas a todas essas questões forem demonstradas cientificamente poderemos conduzir de forma adequada o tratamento por exercício na OA. Uma tentativa de auxiliar na prescrição de exercícios para OA de quadril e joelho, fundamentada no nível de evidência, foi realizada pelo consenso $\mathrm{MOVE}^{(15)}$. As recomendações foram baseadas na opinião de especialistas e evidências em pesquisas $^{(9)}$, porém a eficiência do modelo proposto ainda não foi verificada.

Em vista da necessidade da prática baseada em evidência no tratamento para a OA esta revisão sistemática tem como objetivo elucidar a função e efetividade dos exercícios físicos no tratamento conservador da OA de quadril.

\section{PROCEDIMENTOS DE COLETA}

O levantamento bibliográfico foi realizado nas bases de dados PUBMED, LILACS e MEDLINE referentes às publicações de artigos de ensaio clínico aleatório controlado dos últimos dez anos (julho/2005-julho/1995), utilizando-se a relação entre as palavras-chave osteoarthritis, hip, exercise.

Dezoito artigos resultaram da busca com os critérios supracitados, sendo que todos passaram por análise de resumo por um único avaliador e foram selecionados aqueles que tinham os seguintes critérios de inclusão:

- investigação da relação entre a prática de exercício com a OA de quadril;

- processo de amostragem aleatória dos pacientes; - comparação entre grupo de prática de exercícios e grupocontrole ou grupo com outro tipo de intervenção; - para estudos relacionados com cirurgia necessidade de intervenção antes da cirurgia.

Os artigos que apresentaram estes critérios tiveram seus conteúdos revisados na íntegra e passaram por análise crítica e confrontada com outras publicações sobre o assunto. 


\section{RESULTADOS}

Foram excluídos 8 estudos dos 18 analisados pelo resumo por não corresponderem à relação buscada da prática de exercícios com a OA de quadril. Os estudos excluídos abordavam: utilização de amostras aleatórias de estudos anteriores, porém sem relação com a prática de exercício $^{(19,20)}$; utilização do processo de aleatoriedade para os clínicos e não para os pacientes ${ }^{(21)}$; tratamento envolvendo exercício somente no pós-operatório ${ }^{(22,23)}$, uso de estudo randomizado anterior para comparação com estudo atual de implementação de prática baseada em evidência ${ }^{(24)}$; estudo sem grupo-controle ${ }^{(25)}$ e pesquisa de automanejo da OA baseada em orientações, sem programa específico de exercícios ${ }^{(26)}$.

Foram selecionados dez artigos para a revisão na íntegra e após análise do conteúdo foram identificadas três categorias: a) exercícios específicos para OA de quadril $(\mathrm{n}=3)$; b) exercícios para OA de quadril e joelho $(\mathrm{n}=4)$ e c) exercícios para pré e pós-cirurgia de ATQ decorrente de OA $(n=3)$.

Os dez estudos incluídos fornecem dados de um total de 953 indivíduos com ao, que foram divididos em grupo de terapia por exercício $(\mathrm{n}=503)$ e grupo-controle $(\mathrm{n}=450)$.

\section{EXERCÍCIOS ESPECÍFICOS PARA OA DE QUADRIL}

Os estudos específicos para OA de quadril seguiram os critérios para ensaios clínicos aleatórios controlados e obtiveram suas amostras utilizando critérios diagnóstico e radiológico dividindo-as em grupo controle e experimental ${ }^{(10,27,28)}$. No entanto, dois dos estudos ${ }^{(27,28)}$ optaram por analisar a prática de exercícios como sendo o grupo-controle (terapia usual) versus o grupo experimental composto por uma nova modalidade terapêutica conservadora.

A maioria das amostras era composta por mulheres com idade acima de $65 \operatorname{anos}^{(10,27,28)}$. Apenas o estudo de Haslam ${ }^{(28)}$ tinha amostra considerada pequena, isto é, composição menor que 30 indivíduos em cada grupo.

Os estudos avaliaram o impacto dos exercícios nos aspectos: dor pela Escala Visual Analógica (EVA) ${ }^{(10,27)}$; aspecto funcional pelo instrumento Harris Hip Escore $(H H S)^{10,27)}$ e Western Ontario and MacMaster Universities Osteoarthritis Index (WOMAC modificado) ${ }^{(28)}$; qualidade de vida pelo Helth Related Quality of Life ${ }^{(10)}$ e Short Form $36(S F 36)^{(27)}$; desempenho funcional pelo Time $U p$ \& Go Test (TUGT), sentar e levantar, flexibilidade dedo chão ${ }^{(10)}$ e teste de caminhada ${ }^{(10,27)}$; avaliação subjetiva por escala de Likert ${ }^{(27)}$ e pelo Sickness Impact Profile $(S I P)^{(10)} \mathrm{e}$ avaliação da amplitude de movimento articular - amplitude de movimento (ADM) ${ }^{(27)}$.

As intervenções propostas variaram quanto ao método, tempo, duração e tipo de terapia, estando expostas no Quadro 1. O tempo de análise do efeito da intervenção foi diversificado, sendo analisados os períodos de pré e pós-intervenção imediato ${ }^{(10,27,28)}$, seguimento após dois ${ }^{(28)}$, três ${ }^{(10,27)}$ e seis meses do término da terapia ${ }^{(27)}$.

QUADRO 1

RELAÇÃO DOS ARTIGOS DE ENSAIO CLÍNICO ALEATÓRIO COM INTERVENÇÃO POR EXERCíCIOS PARA OA DE QUADRIL E SUAS PROPOSTAS DE TRATAMENTO

\begin{tabular}{|c|c|c|c|c|c|}
\hline Estudo & Grupo experimental & Grupo controle & Intensidade & Duração & Conclusão \\
\hline Tak et al ${ }^{(10)}$ & $\begin{array}{l}\text { - fortalecimento muscular * } \\
\text { - caminhada * } \\
\text { - exercícios para casa \# } \\
\text { - orientações ergonômicas \# } \\
\text { - orientações nutricionais \# }\end{array}$ & Sem intervenção & $\begin{array}{l}1 \mathrm{vez} / \mathrm{semana} \\
60 \text { minutos }\end{array}$ & 8 semanas & Benéfico \\
\hline Hoeksma et al(27) & $\begin{array}{l}\text { - terapia manual \# } \\
\text { - alongamento \# } \\
\text { - educação \# }\end{array}$ & $\begin{array}{l}\text { - fortalecimento muscular * } \\
\text { - exercícios ativos/passivos * } \\
\text { - alongamento* } \\
\text { - treino de marcha * } \\
\text { - educação * } \\
\text { - exercício para casa \# }\end{array}$ & $\begin{array}{l}2 \text { vezes/semana } \\
25 \text { minutos }\end{array}$ & 5 semanas & $\begin{array}{l}\text { Benéfico para ambos, } \\
\text { porém a terapia manual } \\
\text { mostrou melhores } \\
\text { resultados do que os } \\
\text { exercícios }\end{array}$ \\
\hline Haslam (28) & - acupuntura \# & $\begin{array}{l}\text { - exercícios para casa \# } \\
\text { - educação \# }\end{array}$ & $\begin{array}{l}\text { Experimental: } 1 \mathrm{vez} / \text { semana } \\
25 \text { minutos } \\
\text { Controle: } 1 \text { vez/cada duas semanas } \\
30 \text { minutos }\end{array}$ & 6 semanas & $\begin{array}{l}\text { Benéfico para a intervenção } \\
\text { com acupuntura em relação } \\
\text { aos exercícios }\end{array}$ \\
\hline
\end{tabular}

* tratamento em grupo / \# tratamento individual 
Hoeksma et a ${ }^{(27)}$ obtiveram melhora da dor e ADM com a prática de exercícios. No entanto, a terapia manual mostrou resultados superiores com melhora em $81 \%$ contra $50 \%$ dos casos da terapia por exercícios. No estudo de Tak et $a^{(10)}$, houve melhora estatisticamente significante do grupo experimental em relação ao controle da dor e no HHS e no pós-teste imediato e após três meses na dor, SIP e TUGT. Já o estudo de Haslam ${ }^{(28)}$ não teve melhora com a prática de exercício no WOMAC em nenhuma das avaliações em relação ao pré-tratamento, enquanto que a acupuntura revelou melhora estatisticamente significante comparada ao pré-tratamento e ao grupo de exercício. No grupo de intervenção por acupuntura 31\% dos pacientes reduziram o uso de analgésicos ao final do tratamento, contra $25 \%$ dos pacientes do grupo por exercício, porém sem significância em relação à amostra total.

\section{EXERCÍCIOS PARA OA DE QUADRIL E JOELHO}

Todos os estudos foram compostos por amostras aleatórias divididas em grupo-controle e experimental, sendo que na pesquisa de Foley, Halbert e Croft ${ }^{(29)}$ houve três grupos (grupo de hidroterapia, grupo de exercício, grupo-controle). No entanto, nenhum dos estudos separou os pacientes quanto ao diagnóstico de OA de quadril ou joelho.

Os estudos de Van Barr et al ${ }^{30,31)}$ utilizaram a mesma amostra e intervenção, tendo como objetivo a avaliação do impacto dos exercícios em curto prazo e longo prazo, respectivamente. Todas as amostras foram compostas por mais de 30 indivíduos em cada grupo e com maioria feminina, acima de 65 anos de idade.

Os programas de exercícios propostos estão apresentados no Quadro 2. As avaliações foram feitas pré-intervenção, pós-intervenção ${ }^{(29,30)}$, pós-intervenção de 24 semanas $^{(31,32)}$ e pós-intervenção de 36 semanas $^{(31)}$.

Os estudos tiveram como objetivo analisar os efeitos de um programa de exercícios nos seguintes aspectos: dor pela $\mathrm{EVA}^{(30,31,32)}$; aspecto funcional pelo $\mathrm{WOMAC}^{(29)}$; força muscular pela força de preensão palmar ${ }^{(30,31,32)}$ e de quadríceps $^{(29,32)} ; \mathrm{ADM}^{(30,31,32)}$; qualidade de vida pelo SF-36 ${ }^{(29)} \mathrm{e}$ $\mathrm{EVA}^{(32)}$; desempenho em tarefas por videotape $\mathrm{e}^{(30,31)}$ e pelo instrumento Adelaide Activities Profile ${ }^{(29)}$ e marcha ${ }^{(29,32)}$.

QUADRO 2

RELAÇÃO DOS ARTIGOS DE ENSAIO CLÍNICO ALEATÓRIO COM INTERVENÇÃO POR EXERCÍCIOS PARA OA DE QUADRIL E JOELHO E SUAS PROPOSTAS DE TRATAMENTO

\begin{tabular}{|c|c|c|c|c|c|}
\hline Estudo & Grupo experimental & Grupo controle & Intensidade & Duração & Conclusão \\
\hline Foley et al (29) & $\begin{array}{l}\text { Grupo Hidroterapia } \\
\text { - caminhada na água * } \\
\text { - fortalecimento muscular * } \\
\text { Grupo de Exercícios } \\
\text { - bicicleta * } \\
\text { - fortalecimento muscular * }\end{array}$ & - telefonemas \# & $\begin{array}{l}3 \text { vezes/semana } \\
30 \text { minutos }\end{array}$ & 6 semanas & $\begin{array}{l}\text { Benéfico para ambas as } \\
\text { intervenções }\end{array}$ \\
\hline Van Barr et $a l^{(30)}$ & $\begin{array}{l}\text { - fortalecimento muscular \# } \\
\text { - mobilidade \# } \\
\text { - coordenação \# } \\
\text { - exercícios funcionais \# } \\
\text { - adaptações \# } \\
\text { - exercícios para casa \# } \\
\text { - educação \# }\end{array}$ & - educação \# & $\begin{array}{l}1 \text { a } 3 \text { vezes/ semana } \\
\text { dependendo da } \\
\text { intensidade da dor } \\
30 \text { minutos }\end{array}$ & 12 semanas & Benéfico \\
\hline $\begin{array}{l}\text { Hopman-Rock e } \\
\text { Westhoff }{ }^{(32)}\end{array}$ & $\begin{array}{l}\text { - educação* } \\
\text { - exercícios para casa* } \\
\text { - exercícios de aquecimento* } \\
\text { - exercícios ativos* } \\
\text { - exercícios resistidos* } \\
\text { - relaxamento* }\end{array}$ & Sem intervenção & $\begin{array}{l}1 \text { vez/semana } \\
2 \text { horas }\end{array}$ & 6 semanas & $\begin{array}{l}\text { Razoavelmente } \\
\text { benéfico }\end{array}$ \\
\hline Van Barr et al(31) & $\begin{array}{l}\text { - fortalecimento muscular \# } \\
\text { - mobilidade \# } \\
\text { - coordenação \# } \\
\text { - exercícios funcionais \# } \\
\text { - adaptações \# } \\
\text { - exercícios para casa \# } \\
\text { - educação \# }\end{array}$ & - educação \# & $\begin{array}{l}1 \text { a } 3 \text { vezes/ semana } \\
\text { dependendo da } \\
\text { intensidade da dor } \\
30 \text { minutos }\end{array}$ & 12 semanas & Benéfico em curto prazo \\
\hline
\end{tabular}

* tratamento em grupo / \# tratamento individual 
Com a mesma amostra, os estudos de Van Barr et $a^{(30,31)}$ demonstraram diferenças estatísticas significantes na diminuição da dor, menor uso de paracetamol, aumento da força muscular e melhora da percepção do paciente ao término da intervenção. Após 24 semanas, permaneceu a melhora da percepção do paciente e as demais medidas não tiveram alterações. Após 36 semanas, nenhuma diferença entre os grupos foi observada. Os resultados revelaram que o efeito dos exercícios declina ao longo do tempo.

Os resultados do estudo de Foley et $a^{(29)}$ demonstraram melhora significante da força de quadríceps do grupo de exercícios e hidroterapia em relação ao grupo-controle para o lado esquerdo e somente do lado direito no grupo de exercícios em comparação ao controle. Não houve diferença entre os grupos e pós-intervenção no WOMAC e no Adelaide Activities Profile. A distância percorrida foi maior no grupo de hidroterapia do que no grupo-controle, e esta diferença foi significante. Em relação à velocidade, esta foi maior no grupo de exercícios do que no controle. Os autores concluíram que existem ganhos funcionais em ambas as terapias comparadas com o grupo-controle.

No estudo de Hopman-Rock e Westhoff ${ }^{(32)}$ foi observado melhora significante na dor, qualidade de vida, força muscular de extensão de joelho, auto-eficácia, peso e conhecimento sobre a própria doença.

Somente no estudo de Van Barr et $a^{(30)}$ e Van Barr et al ${ }^{31)}$ foram feitas análises entre a diferença nos resultados quanto ao paciente ser portador de OA de quadril ou joelho.

\section{EXERCÍCIOS PRÉ E PÓS-CIRURGIA DE OA DE QUADRIL}

Os aspectos avaliados quanto ao impacto dos exercícios em pacientes submetidos a ATQ foram: dor pela $\mathrm{EVA}^{(33)}$; força muscular ${ }^{(34)}$, aspecto funcional pelo $\mathrm{HHS}^{(33)} \mathrm{e}$ WOMAC $^{(34)} ; \mathrm{ADM}^{(33,34)}$ e parâmetros da marcha ${ }^{(35)}$.

As intervenções propostas estão descritas no Quadro 3. Wang, Gilbey e Ackland ${ }^{(35)}$ e Gilbey et a ${ }^{(34)}$ utilizaram no pré e pós-cirúrgico a mesma intervenção em diferentes amostras, enquanto no estudo de Gocen et al ${ }^{(33)}$ somente foi feita intervenção no pré-cirúrgico. As avaliações quanto à eficácia do tratamento foram realizadas pré-intervenção, pré-cirurgia, pós-cirurgia de três semanas, pós-cirurgia de 12 semanas e pós-cirurgia de 24 semanas $^{(34,35)}$ e pré-intervenção, pré-cirurgia, pós-cirurgia, pós-cirurgia de 3 meses e pós-cirurgia de 24 meses $^{(33)}$.

QUADRO 3

RELAÇÃO DOS ARTIGOS DE ENSAIO CLÍNICO ALEATÓRIO COM INTERVENÇÃO POR EXERCÍCIOS PARA O PRÉ E PÓS-CIRURGIA OA DE QUADRIL E SUAS PROPOSTAS DE TRATAMENTO

\begin{tabular}{|c|c|c|c|c|c|}
\hline Estudo & Grupo experimental & Grupo controle & Intensidade & Duração & Conclusão \\
\hline Gocen et $a l^{(33)}$ & $\begin{array}{l}\text { - fortalecimento muscular * } \\
\text { - alongamento* } \\
\text { - educação * }\end{array}$ & Sem intervenção & $\begin{array}{l}3 \text { vezes/semana em casa } \\
\text { avaliações a cada duas } \\
\text { semanas na clínica }\end{array}$ & $\begin{array}{l}8 \text { semanas de } \\
\text { pré-operatório }\end{array}$ & Sem benefícios \\
\hline Gilbey et $a \iota^{(34)}$ & $\begin{array}{l}\quad \text { Pré-operatório } \\
\text { - hidroterapia * } \\
\text { - bicicleta estacionária * } \\
\text { - fortalecimento muscular* } \\
\text { Pós-operatório } \\
\text { - caminhada \# } \\
\text { - alongamento \# } \\
\text { - mobilidade em água\# }\end{array}$ & $\begin{array}{l}\text { - orientações rotineiras } \\
\text { dadas no hospital \# }\end{array}$ & $\begin{array}{l}2 \text { sessões supervisionadas e } \\
2 \text { sessões em casa por semana } \\
60 \text { minutos } \\
30 \text { minutos }\end{array}$ & $\begin{array}{l}8 \text { semanas de } \\
\text { pré-operatório } \\
3-24 \text { semanas de } \\
\text { pós-operatório }\end{array}$ & Benéfico \\
\hline $\begin{array}{l}\text { Wang, Gilbey, } \\
\text { Ackland( }^{(35)}\end{array}$ & $\begin{array}{l}\quad \text { Pré-operatório } \\
\text { - hidroterapia* } \\
\text { - bicicleta estacionária* } \\
\text { - fortalecimento muscular * } \\
\text { Pós-operatório } \\
\text { - caminhada \# } \\
\text { - alongamento \# } \\
\text { - mobilidade em água\# }\end{array}$ & $\begin{array}{l}\text { - orientações rotineiras } \\
\text { dadas no hospital \# }\end{array}$ & $\begin{array}{l}2 \text { sessões supervisionadas e } \\
2 \text { sessões em casa por semana } \\
60 \text { minutos } \\
60 \text { minutos }\end{array}$ & $\begin{array}{l}8 \text { semanas de } \\
\text { pré-operatório } \\
\text { 3-24 semanas de } \\
\text { pós-operatório }\end{array}$ & Benéfico \\
\hline
\end{tabular}

* tratamento em grupo / \# tratamento individual 
A amostra de Wang, Gilbey e Ackland ${ }^{(35)}$ foi pequena, com menos de 30 participantes em cada grupo. Somente o estudo de Gocen et al ${ }^{33)}$ teve uma média de idade inferior a 60 anos. Nenhum critério diagnóstico ou radiológico foi utilizado para verificar a gravidade da doença nos indivíduos dos estudos.

Nos aspectos da marcha, foram verificadas melhoras significantes no grupo de exercícios em relação ao controle na velocidade e cadência em todas as avaliações pós-cirurgia, no comprimento do passo após três semanas de cirurgia e na distância em 24 semanas ${ }^{(35)}$. Na avaliação funcional pelo WOMAC, força muscular e amplitude de movimento houve uma melhora significante em todas as avaliações em relação aos dados pré-intervenção e do grupo-controle ${ }^{(34)}$. Assim, estes dois estudos ${ }^{(34,35)}$ revelaram que o mesmo programa de exercícios foi bem tolerado e efetivo para a recuperação precoce após intervenção cirúrgica, bem como a longo prazo. Já no estudo de Gocen et $a^{(33)}$ os resultados mostraram uma melhora significante do grupo experimental, somente logo após a cirurgia quanto ao HHS e na recuperação precoce dos movimentos, porém estes resultados não foram mantidos a longo prazo, concluindo os autores que um programa de exercício não trás benefícios a longo prazo para pacientes submetidos a ATQ.

\section{DISCUSSÃO}

A revisão sistemática de ensaios aleatórios controlados para verificar os benefícios da terapia por exercício na OA de quadril, revelou que sua eficácia como modalidade de tratamento conservador ainda é bastante controversa, já que dos dez estudos três ${ }^{(28,31,33)}$ apresentaram limitações dos benefícios dos exercícios nas amostras. Em uma revisão de ensaios clínicos aleatórios controlados para verificar os benefícios da terapia por exercício na OA de joelho, foram analisados 16 estudos revelando que a intervenção por exercício tem pelo menos efeito imediato moderado na redução da dor e pequeno na função física ${ }^{(13)}$. Esta mesma revisão tinha como objetivo analisar também a OA de quadril, porém não foi possível, em vista do pequeno número de pesquisas. Ainda é escasso o número de estudos clínicos de intervenção conservadora na OA de quadril, comparado com joelho, e este número torna-se ainda menor se pensarmos que quatro dos estudos aqui analisados agruparam estes dois tipos diferentes de OA.

O impacto dos exercícios é avaliado principalmente nos aspectos de dor e função ${ }^{(12)}$, achados estes semelhantes aos da presente revisão. Importante ressaltar que a dor foi a única dimensão avaliada sistematicamente por todos os estudos e por meio do mesmo instrumento de medida, a EVA. Apesar de a maioria dos instrumentos ser reconhecidos na literatura, uma maior uniformidade poderia favorecer a avaliação dos resultados como um todo.

Em relação às propostas terapêuticas várias modalidades de terapia, tipos e duração foram utilizadas. Esta falta de especificação do programa executado talvez seja a maior dificuldade em se obter elevado nível de evidência para a prática clínica. As terapias usadas em todos os artigos envolveram diversos tipos de exercícios em conjunto, não sendo possível revelar se é a combinação ou um tipo específico que deve ser prescrito ou evitado. $\mathrm{Na}$ área de OA de joelho, já existe bom nível de evidência da prática aeróbica e de treinamento muscular, enquanto que para o quadril ainda não é possível obter essas evidências, pelo reduzido número de estudos ${ }^{(15)}$. Também não houve consenso sobre a modalidade de terapia individual ou em grupo.

Nem todos os estudos seguiram rigorosamente critérios de diagnóstico específicos para OA para selecionar os indivíduos, sendo este um fator que pode limitar a terapia por exercício em vista da não existência ou gravidade exacerbada da doença. O ideal seria verificar até que grau da doença os exercícios são benéficos.

A maioria dos estudos apresentou bom tamanho de amostra e as idades foram concentradas na mesma faixa etária, o que ajuda a homogeneizar os resultados. Contudo é importante ressaltar que o estudo de Gocen et a ${ }^{(33)}$ foi o único com amostra de faixa etária inferior a 60 anos e também o que relatou não haver benefícios com a terapia por exercício na recuperação de ATQ. Em geral, indivíduos mais jovens apresentam nível funcional alto e se recuperam mais rapidamente de cirurgias comparados com idosos, o que pode ter influenciado os resultados apresentados.

Nos estudos com exercícios para OA de quadril e joelho, não houve diferença no tratamento nem na avaliação separada dos dois tipos de pacientes presentes na amostra, podendo trazer viés aos resultados dos estudos. Devem ser considerados, separadamente, os aspectos de cada local afetado pela doença para prover um tratamento específico para suas particularidades, além do que o resultado pode ser mascarado pelo bom desempenho dos portadores daquele tipo de OA mais responsivo ao tratamento oferecido.

É importante ressaltar que nem sempre é possível melhorar, principalmente em pacientes com OA grave e em pacientes com idade mais avançada, mas é preciso identificar se existe manutenção do quadro, pois este também é um meio de tratar, isto é, impedir a progressão da doença e das 
incapacidades associadas a ela. Um programa de exercícios não precisa ser somente curativo. Ele pode prevenir perdas de força muscular, de realização das atividades diárias, da $\mathrm{ADM}$, promover o controle da dor e evitar o estabelecimento de deformidades, sendo estas metas valiosas para o profissional que trabalha com pacientes com osteoartrite ${ }^{(36)}$.

\section{CONSIDERAÇÕES FINAIS}

Embora, a maioria dos estudos tenha demonstrado resultados com a prática de exercícios no tratamento conservador da OA de quadril, ainda não é possível determinar o alcance desta intervenção no quadro geral da doença e para

\section{REFERÊNCIAS}

1. Brooks PM: Impact of osteoarthritis on individuals and society: how much disability? Social consequences and health economic implications. Curr Opin Rheumatol 14: 573-7, 2002.

2. Buckwalter JA, Stanish WD, Rosier RN, Schenck RC, Dennid DA, Coutts RD: The increasing need for nonoperative treatment of patients with osteoarthritis. Clin Orthop Relat Res 385: 3645,2001 .

3. Lohmander LS: What can we do about osteoarthritis? Arthritis Res 2: 95-100, 2000.

4. Breedveld FC: Osteoarthritis: the impact of a serious disease. Rheumatology 43: i4-i8, 2004.

5. Arokoski MH, Haara M, Heikki JH, Arokoski JP: Physical function in men with and without hip osteoarthritis. Arch Phys Med Rehabil 85: 574-81, 2004.

6. Sims K: The development of hip osteoarthritis: implications for conservative management. Man Ther 4: 127-35, 1999.

7. Felson DT: Epidemiology of hip and knee osteoarthritis. Epidemiol Rev 10: 1-24, 1998.

8. Reijman M, Hazes JMW, Pols HAP, Bernsen RMD, Koes BW, Zeinstra SMA: Validity and reliability of three definitions of hip osteoarthritis: cross sectional an longitudinal approach. Ann Rheum Dis 63: 1427-33, 2004.

9. Bennell K, Hinman R: Exercise as a treatment for osteoarthritis. Curr Opin Rheumatol 17: 634-40, 2005.

10. Tak E, Staats P, Hespen AV, Hopman-Rock M: The effects of an exercise program for older adults with osteoarthritis of the hip. J Rheumatol 32: 1106-1113, 2005.

11. Buckwalter JA, Saltzman C, Brown T: The impact of osteoarthritis. Clin Orthop Relat Res 427S: S6-S15, 2004.

12. Minor MA: Impact of exercise on osteoarthritis outcomes. J Rheumatol 31: 81-6, 2004.

13. Fransen M, McConnell S, Bell M: Therapeutic exercise for people with osteoarthritis of the hip or knee-asystematic review. J Rheumatol 29: 1737- 45, 2002.

14. Van Barr ME, Assendelft WJJ, Decker J, Oostenforp RAB, Bijlsma JWJ: Effectiveness of exercise therapy in patients with osteoarthritis of the hip or knee: a systematic review of sua progressão. A escassez de estudos controlados aleatórios e as diferentes metodologias nos estudos existentes dificultam o consenso sobre que tipo, intensidade e duração da terapia por exercício na $\mathrm{OA}$ de quadril. A falta de subsídios científicos sobre os exercícios na OA de quadril, expostos nesta revisão, abre um campo de estudo fértil, que possibilita ações de baixo custo e grande impacto social. Sendo assim, são necessários mais estudos nesta área e maior especificidade da intervenção por exercício para que as terapias que revelem bons resultados possam ser compreendidas e reproduzidas, auxiliando assim a prática baseada em evidência.

Declaramos a inexistência de conflitos de interesse. randomized clinical trials. Arthritis Rheum 42: 1361-9, 1999.

15. Roddy E, Zhang W, Doherty $M$ et al: Evidence-based recommendations for the role of exercise in the management of osteoarthritis of the hip or knee- the MOVE consensus. Rheumatology 44: 67-73, 2004.

16. Coimbra IB, Pastor EH, Greve JMD, Puccinelli MLC, Fuller R, Cavalcanti FS: Consenso brasileiro para o tratamento da osteoartrite (artrose). Rev Bras Reumatol 42: 371-4, 2002.

17. ACR Subcommittee on osteoarthritis: Recommendations for the medical management of osteoarthritis of the hip and knee. Arthritis Rheum 43: 190-191b, 2000.

18. Altman RD, Hochberg MD, Moskowitz RW, Schnitzer TJ: Recommendations for the medical management of osteoarthritis of the hip and knee: 2000 update. Arthritis Rheum 43: 1905-15, 2000 .

19. Hoeksma HL, Van Den Ende Ch, Ronday HK, Heering A, Breedveld FC: Comparison of the responsiveness of the harris Hip Score with generic measures for hip function in osteoarthritis of the hip. Ann Rheum Dis 62: 935-8, 2003.

20. Steultjens MP, Dekker J, Bijlsma JW: Coping, pain and disability in osteoarthritis: a longitudinal study. J Rheumatol 28: 1068-72, 2001.

21. Ravaud P, Giraudeau B, Logeart I et al: Management of osteoarthritis $(\mathrm{OA})$ with an unsupervised home based exercise programme and/ or patient administeres assessment tools. A cluster randomized controlled trial with a $2 \mathrm{X} 2$ factorial design. Ann Rheum Dis 63: 703-8, 2004.

22. Mairl J, Faillenet MAF, Grange $C$ et al: A specific arm-interval exercise program could improve the health status and walking ability of elderly patients after total hip arthroplasty: a pilot study. J Rehabil Med 36: 92-4, 2004.

23. McNally MA, Cooke EA, Mollan RA: The effect of active movement of the foot on venous blood flow after total hip replacement. J Bone Joint Surg Am 79: 1198-201, 1997.

24. Jong ORW, Hopman-Rock M, Tak ECMP, Klazinga NS: An implementation study of two evidence-based exercise and health education programmes for older adults with osteoarthrtis of the knee and hip. Health Educ Res 19: 316-25, 2004.

25. Veenhof C, Dekker J, Bijlsma JWJ, Ende CHMVD: Influence 
of various recruitment strategies on the study population and outcome of a randomized controlled trial involving patients with osteoarthritis of the hip or knee. Arthritis Rheum 53: 375-82, 2005.

26. Heuts PH, Bie R, Drietelaar $M$ et al: Self-management in osteoarthritis of hip or knee: a randomized clinical trial in a primary healthcare setting. J Rheumatol 32: 543-9, 2005.

27. Hoeksma HL, Dekker J, Ronday HK et al: Comparison of manual therapy and exercise therapy in osteoarthritis of the hip: a randomized clinical trial. Arthritis Rheum 51: 722-9, 2004.

28. Haslam R: A comparison of acupuncture with advice and exercises on the symptomatic treatment of osteoarthritis of the hip: a randomized controlled trial. Acupunct Med 19: 19-26, 2001.

29. Foley A, Halbert J, Hewitt T, Croft M: Does hydrotherapy improve strenght and physical function in patients with osteoarthritisa randomized controlled trial comparing a gym based and a hydrotherapy based strengthening programme. Ann Rheum Dis 62: 1162-7, 2003.

30. Van Barr ME, Dekker J, Oostendoro RAB et al: The Effectiveness of exercise therapy in patients with osteoarthritis of the hip or knee: a randomized clinical trial. J Rheumatol 25: 2432-9, 1998.

31. Van Barr ME, Deker J, Oostendorp RAB, Bijl D, Voorn TB, Bijlsma JWJ: Effectiveness of exercise therapy in patients with osteoarthritis of the hip or knee: nine months' follow up. Arthritis Rheum 60: 1123-30, 2001.

32. Hopman-Rock M, Westhoff MH: The effects of a health educational and exercise program for older adults with osteoarthritis for the hip or knee. J Rheumatol 27:1947-54, 2000.

33. Gocen Z, Sen A, Unver B, Karatosun V, Gunal I: The effect of preoperative physiotherapy and education on the outcome of total hip replacement: a prospective randomized controlled trial. Clin Rehabil 18: 353-8, 2004

34. Gilbey HJ, Ackland T, Wang AW, Morton AR, Trouchet T, Tapper J: Exercise improves early functional recovery after total hip arthroplasty. Clin Orthop Relat Res 408: 193-200, 2003.

35. Wang AW, Gibey HJ, Ackland TR: Perioperative exercise programs improve early return of ambulatory function after total hip arthroplasty. Am J Phys Med Rehabil 81: 801-6, 2002.

36. Marques AP, Kondo A: A fisioterapia na osteoartrose: uma revisão da literatura. Rev Bras Reumatol 38: 83-90, 1998. 